\title{
Mecanismos de financiamiento generadores de valor social y económico para la educación universitaria: Un caso Latinoamericano*
}

\author{
Financing mechanisms generating social and economic value for university education: a Latin \\ American case \\ Mecanismos de financiamento gerador de valor social e econômico para a educação \\ universitaria: um caso Latino-Americano
}

DOI: https://doi.org/10.21803/pensam.v17i21-1.274

Olandy Naranjo Rivera

https://orcid.org/0000-0002-3711-2204

Gustavo Gregorutti

https://orcid.org/0000-0002-0488-4179

Carlos William Marín

https://orcid.org/0000-0003-3454-4463

Mario Enrique Vargas Saenz https://orcid.org/0000-0002-1081-3190

¿Cómo citar este artículo?

Naranjo, O., Gregorutti, G., Marín, C. \& Vargas, M. (2018). Mecanismos de financiamiento generadores de valor social y económico para la educación universitaria: Un caso Latinoamericano. Pensamiento Americano, 11(22), 168-183.

DOI: https://doi.org/10.21803/pensam.v11i21-1.274

\begin{abstract}
Resumen
Las limitaciones financieras y los escasos recursos económicos disponibles en las universidades son barreras importantes que los jóvenes encuentran para ingresar y concluir exitosamente con un título universitario en la región Latinoamericana. Dado este problema, esta investigación se centró en dos casos de instituciones de educación universitaria (IES) privadas que implementan mecanismos de financiamiento innovadores, que generan valor social y económico para los estudiantes. Estas instituciones se destacan por incluir en sus enunciados misionales estas dimensiones que buscan disminuir la brecha de desigualdad en el acceso a la educación superior.

La investigación utiliza un enfoque cualitativo con el estudio de dos universidades ubicadas en Perú y Colombia. La recolección de datos se basó en entrevistas a directivos de las IES, en la observación del entorno de estudioy en el análisis de documentose informes financieros institucionales de las dos instituciones.

Los resultados indicaron que las IES desarrollan mecanismos de financiamiento basados en transacciones comerciales y no comerciales. La información obtenida permitió concluir que las unidades de negocio, como generadoras de valor social y económico para los estudiantes y las universidades, son trascendentales para el crecimiento económico y sostenible de la misión institucional.
\end{abstract}

Palabras Clave: Educación superior, mecanismos de financiamiento, creación de valor social y económico.

\begin{abstract}
Financial constraints and the scarce economic resources available in universities are essential barriers that young people find to enter and successfully conclude with a university degree in the Latin American region. Given this problem, this research focused on two cases of private university education institutions (HEIs) that implement innovative financing mechanisms that generate social and economic value for students. These institutions stand out for including in their mission statements these dimensions that seek to reduce the inequality gap in access to higher education.

The research uses a qualitative approach with the study of two universities located in Peru and Colombia. The data collection was based on interviews with managers of HEls, on the observation of the study environment and the analysis of documents and institutional financial reports of the two institutions. The results indicated that HEls develop financing mechanisms based on commercial and non-commercial transactions. The information obtained allowed us to conclude that business units, as generators of social and economic value for students and universities, are transcendental for the economic and sustainable growth of the institutional mission.
\end{abstract}

Key words: Higher education, financing mechanisms, creation of social and economic value.

\footnotetext{
* El artículo está adscrito al proyecto de investigación de la línea Desarrollo Social de la UNAC, titulado: El emprendimiento sustentable, una opción de generación de valor económico y social para las Universidades Confesionales.
} 


\section{Resumo}

As limitações financeiras e os escassos recursos econômicos disponíveis nas universidades são barreiras importantes que os jovens encontram para ingressar e concluir com êxito um título universitário na região Latinoamericana. De esta forma, esta pesquisa se centrou em dois casos de instituições de educação universitária (IES) privadas que implementam mecanismos de financiamento inovadores, que geram valor social e econômico para os estudantes. Estas instituições se destacam por incluir em seus enunciados missionais estas dimensões que buscam diminuir a brecha de desigualdade no acesso à educação superior.

A pesquisa parte de um enfoque qualitativo com o estudo das universidades localizadas no Peru e na Colômbia. A coleta de dados foi baseada em entrevistas a diretivos das IES, na observação do entorno do estudo e nas análises de documentos e informes financeiros das instituições. Os resultados indicaram que as IES desenvolvem mecanismos de financiamento baseados em transações comerciais e não comerciais. A informação obtida permitiu concluir que as unidades de negócios, como geradoras de valor social e econômico para os estudantes e universidades são transcendentais para o crescimento econômico e sustentável da missão institucional.

Palavras Chave: Educação superior, mecanismos de financiamento, criação de valor social e econômico.

\section{Perfil}

Decana de la Facultad de Ciencias Administrativas y Contables de la Corporación Universitaria Adventista (UNAC). Especialización en Gerencia de las Organizaciones. Contadora Pública. onaranjo@unac.edu.co

\section{Perfil}

Máster en Administración Educativa - Universidad Adventista del Plata, Argentina. Profesor de Liderazgo y Educación Superior en Andrews University (Michigan, USA). ggregoru@andrews.edu

\section{Perfil}

Magister en Administración, especialista en gerencia de las organizaciones con énfasis en liderazgo, Administrador de empresas.

\section{Perfil}

Co-autor: Director de EAFIT Sede LLanogrande (Colombia)

\section{Olandy Naranjo Rivera}

Magister en ciencias de la Administración y magister en Administración con acentuación en Mercadotecnia

\section{Gustavo Gregorutti}

Ph.D. en Educación Superior Universidad Humboldt y Ph.D. en Administración Educativa y Liderazgo, Universidad Andrews

\section{Carlos William Marín}

Ph.D en proyectos

Mario Enrique Vargas Saenz 


\section{Introducción}

Debido a la constante reducción de recursos públicos dirigidos a dar soporte a las estructuras educacionales, el financiamiento de la educación superior en América Latina ha sido tema de análisis de los diversos actores políticos y cívicos. Frente a esa situación, emerge la necesidad de proponer y desarrollar mecanismos de financiamiento, acordes con las características del entorno, que fomenten la justicia y la equidad social (Barriga, Cardiel, López, Maldonado \& Mendoza, 1996; Bernheim, 2003; Banco Mundial, 2012). En relación a esta problemática, Alcántar, Arcos y Ocegueda (2008) confirman que esta es una posible salida al problema de escasez:

...han aumentado las dificultades para la creación de infraestructura física y humana que respalde adecuadamente la expansión del servicio educativo, por lo que el incremento de los ingresos propios se ha convertido en una de las alternativas más importantes de las instituciones de educación superior (IES) (p.98).

Organismos internacionales como la UNESCO han manifestado que las IES están en la necesidad de revisar las estrategias de gestión de recursos para incrementar sus ingresos propios, diversificando las fuentes de financiamiento y adoptando prácticas acordes con las necesidades de su entorno, con el fin de responder "a las necesidades de los diferentes componentes de la sociedad" (Bernheim, 2003, p.194).

Las IES consecuentemente necesitan gestionar y adoptar mecanismos de financiamiento alternativos a las matrículas (Casani, Pérez-Esparrells, \& Rodríguez, 2010; De Fanelli, 2007), debido a la escasez de recursos y al incremento en la demanda educativa de la población juvenil. Por lo tanto, es primordial una adecuada asignación y movilización de los recursos financieros en la gestión de las instituciones de educación superior (IES), para contribuir estratégicamente al desarrollo de las diversas misiones sociales y educativas que beneficien a grupos específicos con dificultades financieras para cursar una carrera universitaria.

En este contexto, este trabajo presenta el análisis de dos casos de IES ubicadas en Perú y Colombia, caracterizadas por desarrollar mecanismos innovadores de financiamiento a estudiantes carenciados.

\section{Marco conceptual}

\subsection{Los mecanismos de financiamiento uni- versitarios}

Un mecanismo de financiamiento (MF) es un proceso que moviliza recursos económicos indispensables para el desarrollo de las IES. Según sea el mecanismo de financiación aprobado e implementado, las instituciones pueden llegar a generar impactos sociales de gran cobertura que incluyen la participación de múltiples grupos de interés. Es decir, se busca el cumplimiento de metas estratégicas relacionadas con objetivos sociales así como también actividades financieras específicas que beneficien la institución (Austin, Gutiérrez, Ogliastri, \& Reficco, 2006).

Los mecanismos de financiamiento pueden clasificarse en dos grandes grupos: los que se desarrollan a través del mercado y los que se realizan fuera de él. El primero incluye transacciones comerciales o de inversión, en relación directa o indirecta con el objeto social de la organización. Ejemplos de este tipo de mecanismos son la venta de bienes y servicios al público en general, el cobro de cuotas a beneficiarios, las campañas de marketing de causa, así como los fondos de inversión social

Pensamiento Americano Vol. 11 (22) • 2018 • Julio-Diciembre · Corporación Universitaria Americana · Barranquilla, Colombia • ISSN: $2027-2448$. 
o ambiental (Austin et al., 2006). Los recursos que se producen fuera del mercado, provienen de fuentes de financiamiento como los donativos, las asignaciones de fondos públicos nacionales o extranjeros (Améstica, Gaete, \& Llinas-Audet, 2014), o por las partidas presupuestarias internas de la organización que no son originadas directamente por el objeto social de la institución (Ver tabla 1).

Las IES pueden implementar mecanismos cuyo origen provenga de diversas fuentes de financiamiento, así como también movilizar los recursos a través de limitadas fuentes de operación. Estas decisiones pueden generar una dependencia moderada o significativa, respecto a la sostenibilidad de la organización, con base en el grado de afectación que implique cualquier cambio en el entorno o al interior de la estructura de la organización (Austin et al., 2006).

Las IES pueden implementar mecanismos cuyo origen provenga de diversas fuentes de financiamiento, así como también movilizar los recursos a través de limitadas fuentes de operación. Estas decisiones pueden generar una dependencia moderada o significativa, respecto a la sostenibilidad de la organización, dependiendo del grado de impacto que implique cualquier cambio en el entorno o al interior de la estructura de la organización (Austin et al., 2006).

\subsection{Creación de valor social y económico}

Tradicionalmente, el interés por la generación de valor social se identificaba como una preocupación predominantemente de las organizaciones del tercer sector, tales como: asociaciones, fundaciones, entidades religiosas, cooperativas de iniciativa social, o empresas de inserción (Alter, 2006; Vásquez \& Dávila, 2008; Young, 2006). Por otra parte y en contraposición, los objetivos del sector privado se alineaban preferentemente con la creación de valor económico para obtener lucro (Austin et al., 2006; Molina, Armenteros \& López,

Tabla 1.

Mecanismos y fuentes de financiamiento.

\begin{tabular}{|c|c|c|}
\hline & Mecanismos & Fuentes de financiamiento \\
\hline 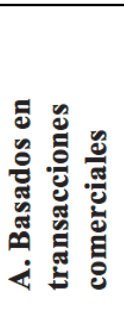 & $\begin{array}{l}\text { A1. Venta de bienes o servicios al } \\
\text { público en general } \\
\begin{array}{l}\text { A2. Cuotas de los beneficiarios } \\
\text { de la institución }\end{array} \\
\text { A3. Marketing de causa }\end{array}$ & $\begin{array}{l}\text { Cartera de clientes involucrados/número } \\
\text { de bienes y servicios ofrecidos } \\
\text { Cartera de beneficiarios con poder } \\
\text { adquisitivo/tipos de cuotas consideradas } \\
\text { Campañas existentes/número de productos o } \\
\text { servicios incluidos en estas }\end{array}$ \\
\hline 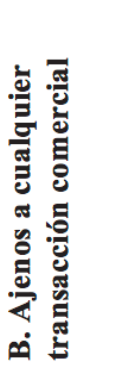 & $\begin{array}{l}\text { B1. Donaciones en efectivo } \\
\text { o especie } \\
\text { B2. Recursos públicos (líneas de } \\
\text { crédito, contratos de provisión de } \\
\text { servicios, contribuciones o subsidios) } \\
\text { B3. Presupuesto interno de empresas }\end{array}$ & $\begin{array}{l}\text { Base de donantes (personales, empresariales, } \\
\text { multilaterales) } \\
\text { Gobiernos extranjeros, nacionales, } \\
\text { estatales y locales } \\
\text { Unidades de negocio o subsidiarias que } \\
\text { forman parte del esquema de financiamiento } \\
\text { (franquicias). }\end{array}$ \\
\hline
\end{tabular}

Fuente: Austin et al. (2006, p.191). 
2014; Porter \& Kramer, 2011). En el entorno actual, la creación de valor social y económico se ha convertido en un asunto que compete a ambos sectores, debido a la creciente importancia que cobra el desarrollo del objeto social de la organización que busca la optimización de los recursos y la inversión en proyectos que apoyen los objetivos de la empresa y de los diferentes grupos de interés (De Benito, 2008; Muñoz, 2013; Méndez \& Peralta, 2014; Díaz \& Fernández, 2016; Díaz \& Castaño, 2013).

Mair y Noboa (2003), explican que una organización puede caracterizarse por la creación simultánea de valor social y económico, en alineación con la misión orientada a generar un cambio social, bajo la estructura de un modelo de negocio autosuficiente. Es así como la propuesta de valor se convierte en el fundamento de las organizaciones. Esto hace que las decisiones estratégicas y sus alcances en la estructura, procesos y asignación de recursos sea determinando por los beneficiarios de la misión de la organización (Austin et al., 2006; Spickett-Jones, Philip, \& Jon, 2004; Young, 2006). La claridad en el proceso de creación de valor social y económico de la empresa determinará complementariamente cómo servir a los clientes, generando diferentes dinámicas para satisfacer las necesidades del público objetivo.

La creación de valor social es el distintivo fundamental de las empresas que están orientadas por el compromiso de contribuir al cambio social y, al mismo tiempo, a la sostenibilidad de la organización (Vásquez \& Dávila, 2008). Por esa razón, los modelos de negocio de esta clase de organizaciones se concentran principalmente en la sostenibilidad financiera para garantizar el desarrollo de la misión organizacional (Novy-Hildesley, 2007). Esto significa que el centro de interés primordial está en cambiar las vidas de los individuos, a través del logro de objetivos socialmente deseables (Austin et al., 2006). El valor económico se hace visible "por la voluntad del cliente en remunerar la provisión del bien recibido con sus propios recursos y el precio resulta un indicador del valor que está capturando" (Austin et al., 2006, p. 286). De esta forma, la generación de valor social es el resultado del intercambio comercial en torno al bien o servicio adquirido por el cliente quien directamente se convierte en la fuente principal de financiación de las empresas sociales. Tradicionalmente se considera que un negocio es rentable cuando el valor creado supera el costo de las actividades que dieron origen al valor entregado al cliente (Lescano, 2011). Así, la empresa puede incrementar el valor económico en la medida que aumenta la satisfacción de las necesidades del cliente, al mismo tiempo que disminuye el uso de los recursos para la generación de un valor específico. La empresa social como un tipo de organización sin ánimo de lucro (Naranjo, 2015), genera excedentes económicos que son reinvertidos nuevamente en el desarrollo del objeto social de la organización. La creación de valor económico en la empresa social va más allá de la producción de riqueza para los propietarios o socios, como lo describe Duque (2007):

No está relacionada o alineada con la perspectiva de la ideología gerencial tradicional cuyo propósito es la maximización del beneficio, la riqueza y del valor para el accionista/ propietario, sino con la reinversión de los beneficios en el negocio mismo y en la comunidad (p.62).

La búsqueda organizacional de la creación simultanea de valor social y económico permite a la empresa generar el ambiente para atraer más inversionistas, que fomentan la producción del valor compartido (Porter \& Kramer, 2011) para promover la misión institucional y satisfacer las necesidades de la co-

Pensamiento Americano Vol. 11 (22) • 2018 • Julio-Diciembre · Corporación Universitaria Americana · Barranquilla, Colombia • ISSN: $2027-2448$. 
munidad con el apoyo estratégico de los diferentes grupos de interés (Terzolo, 2014). Como resultado, la empresa estará en condiciones de dar continuidad a su labor social al mismo tiempo que produce los recursos financieros suficientes para su sostenibilidad a largo plazo (Coraggio, 2011; Demil \& Lecocq, 2009; Nicholls, 2006). Esta acción redunda en la generación de "...beneficios individuales y sociales, representados no sólo en el aumento de la remuneración y el crecimiento de la economía, sino que también augura mejor calidad de vida para las personas que tienen acceso a ella" (Ruiz, Cano, \& Montes, 2008, p.112).

\section{Metodología}

La revisión de literatura planteada anteriormente ayudó a enmarcar la siguiente pregunta de investigación: ¿Qué mecanismos de financiamiento utilizan las universidades para generar valor social y económico a los estudiantes de bajos recursos? Para responder a esta pregunta general, se desarrolló una investigación cualitativa de estudio de casos instrumental de tipo múltiple o colectivo (Stake, 2013). El estudio de caso instrumental es definido por Stake (1998) con las siguientes palabras:

...un tipo de estudio donde el investigador tiene interés en examinar el caso en particular principalmente para brindar una comprensión de una cuestión o para volver a trazar una generalización. El caso es de interés secundario, tiene una función de respaldo y facilita nuestro entendimiento de algo más. El caso es analizado en profundidad, sus contextos son escrutados y sus actividades ordinarias, detalladas, pero todo se debe a que ello nos ayuda a perseguir el interés externo (p.17) .

Para la investigación, el interés externo se enmarca en la necesidad de examinar los mecanismos de financiamiento utilizados por las universidades y reconocer las oportunidades ofrecidas a un grupo de jóvenes con limitaciones económicas para ingresar a la educación superior. A continuación, se describirá brevemente los casos elegidos y las principales características que hacen de estas universidades un estudio de caso pertinente, en la búsqueda de posibles modelos para el financiamiento de la educación en países latinoamericanos.

\subsection{Caso elegido}

Las IES objeto de estudio fueron seleccionadas por poseer una vasta experiencia en la creación de empresas universitarias con el claro propósito misional de facilitar a través de estas figuras empresariales una educación superior accesible a la población de escasos recursos económicos. Ambas instituciones forman parte de un sistema internacional de más de 100 instituciones universitarias de la Iglesia Adventista del Séptimo día y se destacan por proveer un modelo educativo que combina experiencias tanto teóricas como prácticas en la búsqueda de una formación integral.

El primer caso se encuentra en la ciudad de Medellín en Colombia; fundada en 1937. Esta universidad cuenta con un promedio de 1500 estudiantes por año, reclutando jóvenes de los estratos 1, 2 y 3 oriundos de los principales departamentos del país con algunos pocos alumnos internacionales. Esta situación ha demandado de la administración de la institución un mayor esfuerzo para facilitar residencias y espacios laborales para aquellos jóvenes que no se encuentran en condiciones de financiar sus cursos de estudio. Así se han generado diferentes empresas de servicios e industrias para la inserción de los estudiantes. La industria de la IES colombiana genera recursos significativos a partir de las relaciones comerciales sostenidas con grandes plata- 
formas y almacenes de cadena del país. Este hecho facilita la vinculación de los estudiantes en un espacio de experiencia laboral para integrar teoría y práctica en sus puestos de trabajo.

El segundo caso elegido es de Perú. La institución fundada en 1929 cuenta con un promedio de 4.800 estudiantes por año en la sede principal de Lima. El 50\% de la población estudiantil posee los recursos económicos suficientes para estudiar. Entre el 30 y $40 \%$ busca diferentes mecanismos de autofinanciación externos a la universidad, y un promedio del $20 \%$ se apalanca en las oportunidades laborales ofrecidas por la universidad a través de las industrias. Una de las industrias es reconocida en el mercado por la singularidad de sus productos que han experimentado un marcado crecimiento de ventas en los últimos 10 años.

Por lo tanto, cada institución elegida como objeto de estudio posee características similares respecto a su modelo educativo, la creación de empresas universitarias y la misión de facilitar la accesibilidad a la educación superior. De acuerdo con las características del caso elegido y al objetivo de la investigación, se procedió a aplicar técnicas de recolección cualitativas como la entrevista, el análisis documental y la observación

\subsection{Técnicas de recolección de información}

La información recolectada en el proceso de

Tabla 2.

Técnicas y fuentes de información

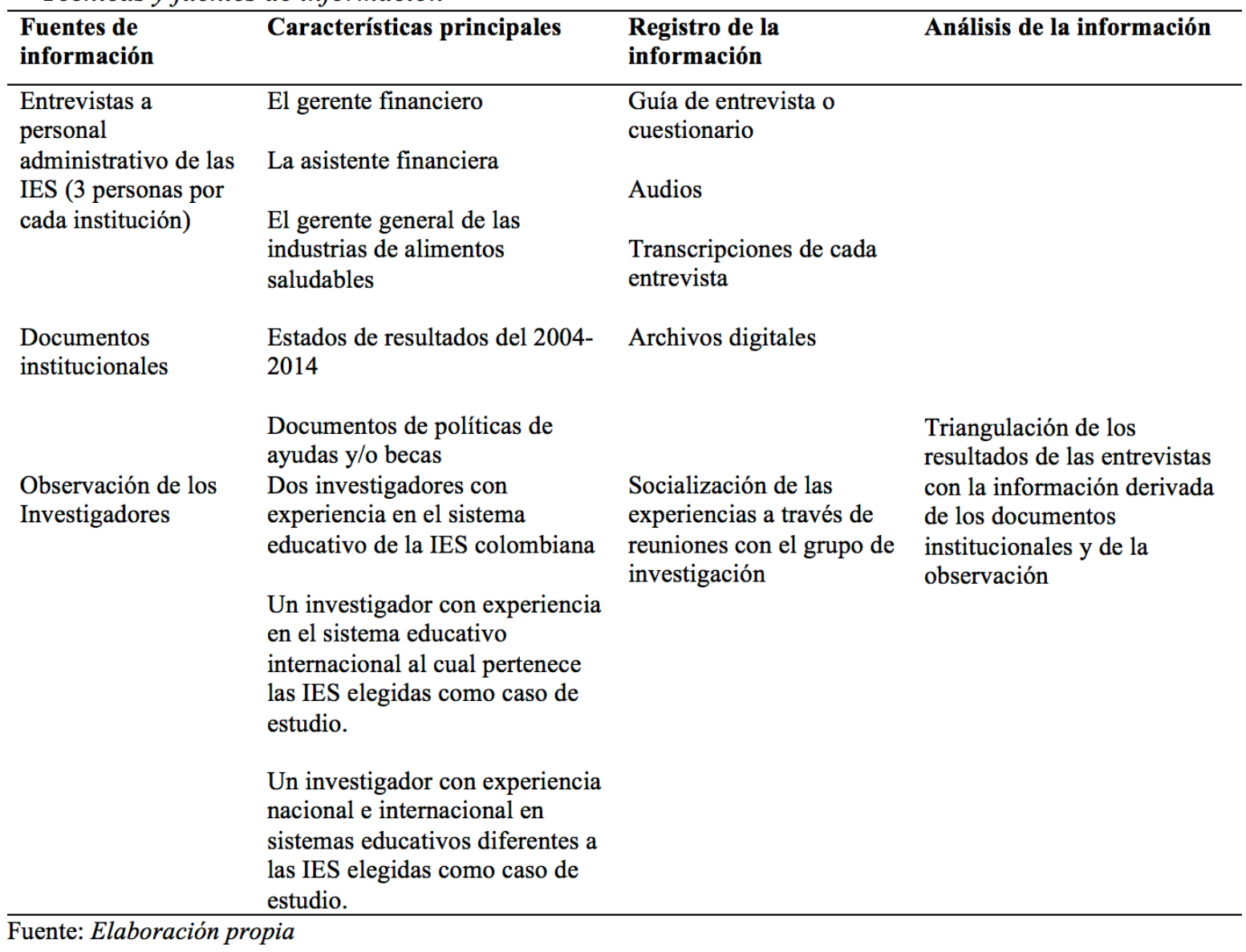

Pensamiento Americano Vol. 11 (22) • 2018 • Julio-Diciembre • Corporación Universitaria Americana • Barranquilla, Colombia • ISSN: $2027-2448$. http://publicaciones.americana.edu.co/index.php/pensamientoamericano/index 
investigación provino básicamente de tres fuentes: Entrevistas, documentos institucionales y observación. En la tabla 2 se describe de manera general las fuentes de información recolectadas en las dos IES.

\section{Resultados}

Los resultados de las entrevistas y de los documentos institucionales, permitieron identificar los principales mecanismos de financiamiento adoptados por las IES, con algunas similitudes y diferencias entre ellas, según las características de cada universidad. A continuación, en la tabla 3 se presenta en forma concreta los principales mecanismos que sirven a la financiación de la institución y de los jóvenes con barreras de ingreso económico a la educación terciaria.

\subsection{MF IES colombiana}

El MF basado en transacciones comerciales de la IES colombiana a partir de la venta del servicio de educación, incluye principalmente las matrículas y otros conceptos que forman parte del proceso como las inscripciones, nivelatorios, supletorios, suficiencias, validaciones, certificaciones y derechos de grado. La institución también ha generado otros MF definidos como unidades de negocio, los cuales contribuyen en primer lugar a suplir servicios internos y en segundo lugar sirve a las demandas de clientes externos. Estas unidades de negocio comprenden: centro de idiomas, academia de música, centro audiovisual, restaurante, minimercado, litografía, taller de confecciones, IPS universitaria e industria de alimentos.

La fuente de ingresos generada por la industria de alimentos es uno de los MF más representativos entre las unidades de negocio respecto a su contribución en el presupuesto de la institución para el año 2015. Así lo expresó el Vicerrector financiero: "los ingresos de la industria son ostensiblemente mucho mayor que los de la universidad. Sólo la industria puede representar hasta el 57 y 60\% de los ingresos de toda la universidad...en este momento de la historia". De acuerdo con las políticas actuales institucionales, del 100\% de las utilidades generadas por la industria de alimentos, el 40\% de los réditos están estipulados para apoyar un $20 \%$ al desarrollo académico, un 10\% al desarrollo de la investigación

Tabla 3.

Mecanismos de financiamiento universitario.

\begin{tabular}{llll}
\hline Institución & $\begin{array}{l}\text { Mecanismo (s) basados en } \\
\text { transacciones comerciales }\end{array}$ & $\begin{array}{l}\text { Mecanismo (s) ajenos a } \\
\text { transacciones comerciales }\end{array}$ \\
\hline IES colombiana & $\begin{array}{l}\text { A1. Venta de servicios de educación } \\
\text { superior (matrículas y enseñanza) y }\end{array}$ & $\begin{array}{l}\text { B1. Donaciones en efectivo } \\
\text { (socios institucionales y de } \\
\text { venta de productos a través de }\end{array}$ & $\begin{array}{l}\text { empleados) } \\
\text { B2. Recursos público- } \\
\text { unidades de negocio }\end{array}$ \\
$\begin{array}{l}\text { A2. Cuotas de los estudiantes } \\
\text { beneficiarios de las becas }\end{array}$ & $\begin{array}{l}\text { (Fondos yobierno local } \\
\text { (Fon) }\end{array}$
\end{tabular}

IES peruana

A1. Venta de servicios de educación superior (matrículas) y venta de productos a través de unidades de negocio
B1. Donaciones en efectivo B2. Recursos públicos del gobierno local.

Fuente: Elaboración propia a partir de Austin et al. (2006).

Pensamiento Americano Vol. 11 (22) • 2018 • Julio-Diciembre · Corporación Universitaria Americana · Barranquilla, Colombia • ISSN: $2027-2448$. 
y el restante a otros proyectos específicos de la institución (Ver Gráfica 1).

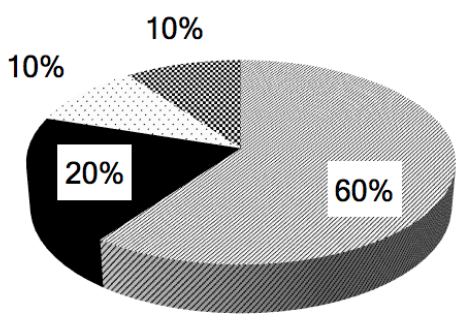

Gráfica 1: Distribución de las utilidades de la industria de alimentos en la IES colombiana.

Fuente: Elaboración propia.

La industria de alimentos como MF, se ha convertido en una fuente de apalancamiento importante para el desarrollo y sostenibilidad de la universidad colombiana y consecuentemente llega a ser un medio de financiamiento importante para los estudiantes que trabajan en dicha industria, porque pueden sufragar los gastos durante el curso de su carrera y al mismo tiempo adquirir una experiencia laboral necesaria para su formación profesional. El Vicerrector financiero de la IES lo confirma con las siguientes palabras: "Estas figuras complementan la educación, y fueron muy bien concebidas...con dos enfoques: uno misional a través de la producción y comercialización de productos sanos, y segundo se están usando como mecanismo de financiación para los estudiantes de bajos recursos." Es decir, además de funcionar como un comercio, esta industria contribuye al desarrollo educativo de los estudiantes.

En relación con el MF basado en las cuotas de los estudiantes beneficiarios de las becas (el porcentaje de contribución que debe aportar el estudiante para optar por el beneficio de la beca), la institución ofrece subvenciones que apuntan a cubrir la vivienda, manutención y costos del semestre de aquellos jóvenes que ingresan a laborar en las diferentes unidades de

negocio o dependencias administrativas de la universidad. De acuerdo con la información recolectada en las entrevistas, aproximadamente el 20\% del total de los estudiantes que ingresan anualmente a la institución son beneficiados con esta clase de financiamiento.

Los MF ajenos a cualquier transacción comercial en la IES colombiana provienen principalmente de dos fuentes: las donaciones y los recursos públicos (Alcántar et al., 2008; De Fanelli, 2007). La primera se origina en los aportes de aliados institucionales quienes buscan subvencionar una parte de los gastos salariales del cuerpo docente y otros proyectos institucionales. Los empleados también realizan donaciones con el propósito de financiar la carrera o la manutención de algunos estudiantes (Casani et al., 2010). Este tipo de MF ha servido para apoyar el valor social de la IES como lo es la educación en dos sentidos, como una fuente generadora de valor económico a la institución y al mismo tiempo a los estudiantes. Por lo tanto, contribuye a potencializar el valor social de la IES, porque permite generar recursos en una doble vía para beneficio no sólo de los clientes de la institución, sino también a la gestión de la administración de la organización (Young, 2006).

La fuente originada en los recursos públicos surge de fondos financiadores de la educación de estudiantes de estratos bajos de la ciudad y que buscan contribuir en la disminución de la brecha en el acceso a la educación superior otorgando becas en IES privadas a esta parte de la población. Este MF, aunque no es preponderante como fuente de financiación para la IES, contribuye a la generación de valor económico para los estudiantes beneficiarios de esta clase de ayuda por parte de entidades públicas como la Alcaldía, la Gobernación o el Estado.

La información financiera facilitada por la IES 
colombiana permitió visualizar el porcentaje de contribución de MF basados en transacciones comerciales (matrículas y enseñanza y unidades de negocio), así como también el de los MF ajenos a cualquier transacción comercial específicamente las donaciones en un periodo comprendido entre el 2004 y el 2014 (Ver Gráfica 2).

Los datos estadísticos recabados entre 2004 a 2014 muestran como del total de las fuentes de financiación de la institución el porcentaje de contribución por concepto de matrículas y enseñanza presenta un comportamiento relativamente constante de un año al otro, tendiendo reiterativamente durante todo el periodo, a ser el 50\% de las fuentes de financiamiento.

Se observa que los mecanismos de tipo comercial, como la contribución por matrículas y enseñanza y la contribución por unidades de negocio, han sido los medios que más aportan a su sostenibilidad. Sin embargo, la financiación por medios no comerciales, como las donaciones, han venido aumentando paulatinamente, pasando de un $12 \%$ a casi a una cuarta parte en el último año.

Si se toma en cuenta que el número de es- tudiantes ha aumentado en un 19,2\% desde 2010 hasta 2014, llegando al 52\% por aporte de matrículas, podría deducirse que tanto el aporte de industrias como de donaciones ha estado aumentando. Sin embargo, se nota que la contribución porcentual de las industrias tiende a disminuir en valor absoluto. Así, se destaca una participación actual de contribuciones no directamente relacionados con el objeto social de la institución, aportes de industrias y por donaciones, correspondientes a casi la mitad de las fuentes de financiación.

\subsection{MF IES peruana}

Los MF basados en transacciones comerciales e identificadas en la IES peruana, operan bajo el mismo modelo de venta de servicios de educación, el cual incluye las matrículas y otras fuentes alusivas a la enseñanza. Referente a las unidades de negocio creadas por esta institución (restaurante, bazar, industria de alimentos, centro audiovisual, imprenta y distribuidora de publicaciones institucionales). Una de las más representativas es la industria de alimentos por su reconocimiento en el mercado y por la contribución financiera al presupuesto total de la universidad actualmente representada en un 30\%, según la información suministrada durante la entre-

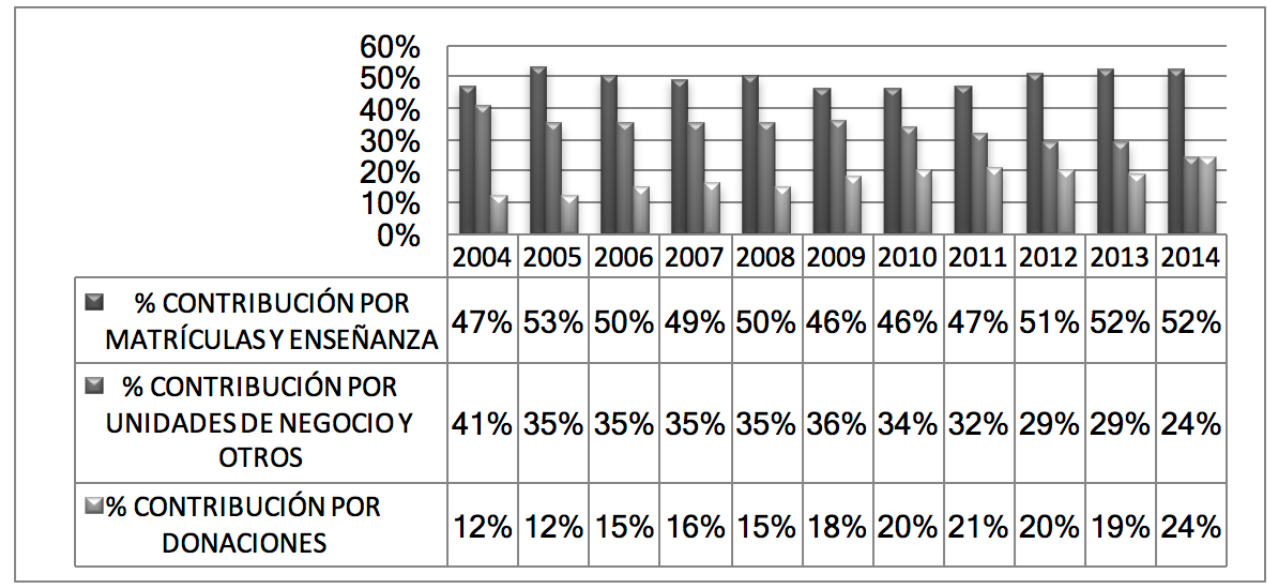

Gráfica 2: Mecanismos de financiamiento IES Colombiana 2004-2014.

Fuente: Elaboración propia. 
vista por el gerente financiero de la IES.

Los datos relacionados con el porcentaje de contribución de esta unidad de negocio en un periodo comprendido entre el año 2004 hasta el 2014 (Ver Gráfica 3), permiten observar variaciones continuas durante todo el periodo, a razón de que en los primeros años el $100 \%$ de la utilidad era direccionada a las funciones y actividades globales de la IES. Sin embargo, por la necesidad de replantear la estrategia y el crecimiento de la industria en el largo plazo, se tomó la decisión de reinvertir la mayor parte de las utilidades en la operación de la industria. Por esa razón, se observa una tendencia a disminuir, notándose que el aporte de la industria se ha reducido hasta llegar aproximadamente a un 30\% de la utilidad total.

En relación con los estudiantes, la industria de alimentos ha sido un punto de apalancamiento para los jóvenes provenientes de familias económicamente limitadas, pero que han considerado este medio como una alternativa para la financiación de la educación de sus hijos, así lo expresó el gerente de la industria universitaria en Perú:
Los padres de familia...saben que el hecho de no tener recursos para mandar a estudiar a sus hijos eso no es excusa para no mandarlos a estudiar, muchos han venido aquí y nos hemos valido de esta oportunidad que la universidad le da y creo que ese es un valor diferencial que tiene la universidad también, el hecho de estar formando profesionales que tienen mucha dificultad para hacerlo.

Desde esa perspectiva las IES generan un valor social como lo es la educación para un grupo de jóvenes con barreras de ingreso, a través de una estrategia productiva que genera rentabilidad y sostenibilidad en el largo plazo (Alter, 2006; Coraggio, 2011; Céspedes, 2009). La administración de las IES y el MF alternativo con base en la creación de unidades de negocio (Pazos, López, González \& Sandiás, 2008); ha suplido las necesidades de los estudiantes que no pueden acceder a una educación superior (Austin et al., 2006; Foncea, Marcuello \& Marcuello, 2012; Vázquez-Maguirre \& Portales, 2014). Por lo tanto, se puede afirmar que la generación de valor social como principio misional de las universidades, ha potencializado el valor económico de la institución (Austin et al., 2006). La administra-

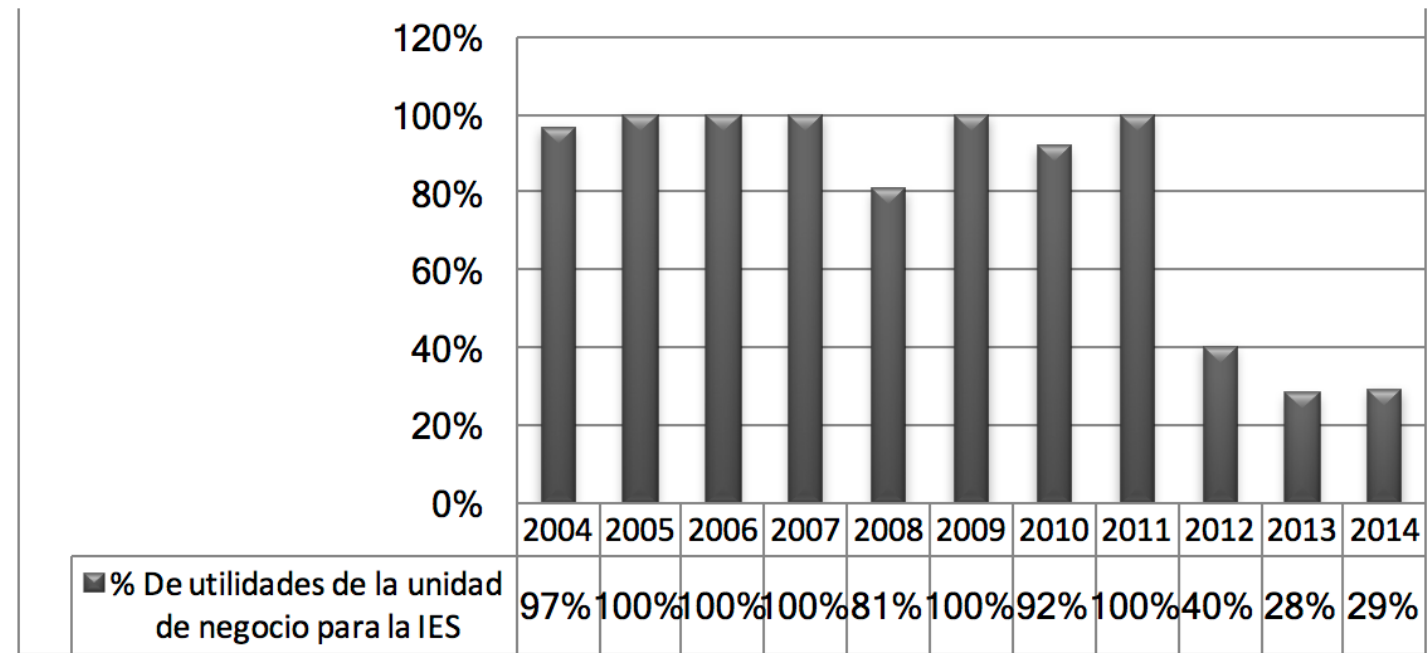

Gráfica 3: Porcentaje de contribución de la industria de alimentos para la IES peruana.

Fuente: Elaboración propia.

Pensamiento Americano Vol. 11 (22) • 2018 • Julio-Diciembre · Corporación Universitaria Americana · Barranquilla, Colombia • ISSN: $2027-2448$. 
ción de la IES peruana también hizo hincapié en cómo el trabajo de los estudiantes a través de la venta de los productos puerta a puerta de la industria de alimentos, ha servido de estrategia de marketing para reforzar la marca de la universidad y de la unidad de negocio en el mercado, debido a que los clientes reconocen el valor social y económico generado por la industria para este grupo de jóvenes. De esta forma lo expresó los administradores de la industria:

...ese joven toca la puerta de la casa entonces cuando llega tocan la puerta de la casa, alguien le abre y ve que es un estudiante, la gente dice no solo estoy consumiendo un producto saludable, sino que estoy ayudando a un joven para que siga estudiando... el estudiante llega con un uniforme, un polo que dice "estudiante emprendedor" con eso llega y obviamente hay un impacto en el público...y gracias creo que a los alumnos, productos $x$ es tan conocido como lo es hasta hoy en día.

Otra unidad de negocio destacada como MF para la IES es la distribuidora de publicaciones institucionales, la cual ha representado una alternativa atractiva para aquellos estudiantes que prefieren subvencionar sus estudios por medio de un trabajo más independiente y arriesgado pero probablemente más productivo, porque no cuentan con un sueldo fijo sino con bonificaciones cuyo valor varían según el nivel de ventas de las publicaciones y que finalmente cubren una parte del valor de la matrícula de la carrera profesional.

El gerente financiero de la IES peruana aseguró que esta fuente de ingreso cumple varios propósitos: proveer recursos al estudiante, generar utilidades para la distribuidora y consecuentemente para la IES, quien finalmente recibe el dinero generado por los jóvenes a través de este tipo de labor. Este MF se ha convertido en un programa bandera para la IES porque vincula laboralmente a un número importante de estudiantes. Así lo explica el gerente financiero de la IES: “...hay más o menos 1000 alumnos trabajando en los tres campus, esta es una de las actividades que en los últimos años ha venido ganando más espacio para darles oportunidades a los chicos para conseguir recursos".

Los MF ajenos a cualquier transacción comercial como lo son las donaciones, aunque no es una fuente de ingreso significativa para la IES, debido a que representa entre el $0 \%$ y el $4 \%$ de los ingresos totales, también contribuye mínimamente a la financiación del objeto social de la institución. Su origen radica en los aportes de aliados institucionales que destinan una partida para el desarrollo de proyectos específicos en función de la educación.

Los recursos públicos se han convertido para la IES en otro MF facilitador para insertar a los jóvenes en la educación terciaria, a través del plan de Modalidad Formativa Laboral creado por el gobierno peruano para incentivar en las empresas la vinculación de jóvenes sin experiencia laboral. Es así como la IES ha aprovechado este mecanismo para ofrecer a los estudiantes una oportunidad económica para el financiamiento de su carrera, insertándolos laboralmente en los diferentes departamentos o en las unidades de negocio que operan al interior de la institución. La administración de la IES considera que el número de estudiantes que se benefician de este MF son pocos, aunque se reconoce como otra forma de ayuda para esta población de acuerdo con la siguiente declaración:

...en realidad no son muchos los que tengo en el reporte por ejemplo para el año 2013 teníamos 148 alumnos trabajando en las diversas áreas, en el 2014 la cifra era de 115, este año (2015) el número de estudiantes es de 157...Es un plan de gobierno esto está dentro de un 
marco legal...y es una forma de ayudar dándoles trabajo a los chicos.

Los MF implementados por la administración en la IES peruana, han cumplido el propósito para la cual fueron creados: generar valor social y económico para la población juvenil y para la institución. En la ilustración 4 se puede visualizar el nivel de contribución porcentual que ha generado los principales MF de la IES entre los años 2004 y 2014.

Los datos estadísticos recolectados correspondientes al periodo 2004-2014 muestran que del total de las fuentes de financiación de la institución el porcentaje de contribución por concepto de matrículas y enseñanza ha estado siendo fortalecido por otros mecanismos de financiamiento en los últimos años. De ser el 75\% de las fuentes de financiamiento, actualmente su tendencia es a acercarse al $50 \%$.

Se observa que los mecanismos de tipo comercial, como la contribución por matrículas y enseñanza y la contribución por unidades de negocio, han sido los medios que más aportan a su sostenibilidad, con una participación mayor al 95\%.

Aunque el aporte de industrias se ha reducido como porcentaje de aporte, sin embargo, ha crecido en su participación como fuente de financiamiento, hasta llegar a ser un $43 \%$. Las donaciones, no siendo todavía muy significativas, muestran una tendencia a crecer.

Así, se muestra una participación actual de contribuciones no directamente relacionados con el objeto social de la institución, aportes de industrias y por donaciones, acercándose a la mitad de las fuentes de financiación.

\section{Conclusiones}

Como se presentó en este estudio, los dos casos de IES tienen un modelo de financiación innovador que va más allá del tradicional ingreso de matrículas. Esto es particularmente relevante en el contexto de la creciente cantidad de universidades privadas en la región de Latinoamérica (Rama, 2012). Las dos instituciones presentadas aquí aportan una perspectiva que combina un equilibrio finan-

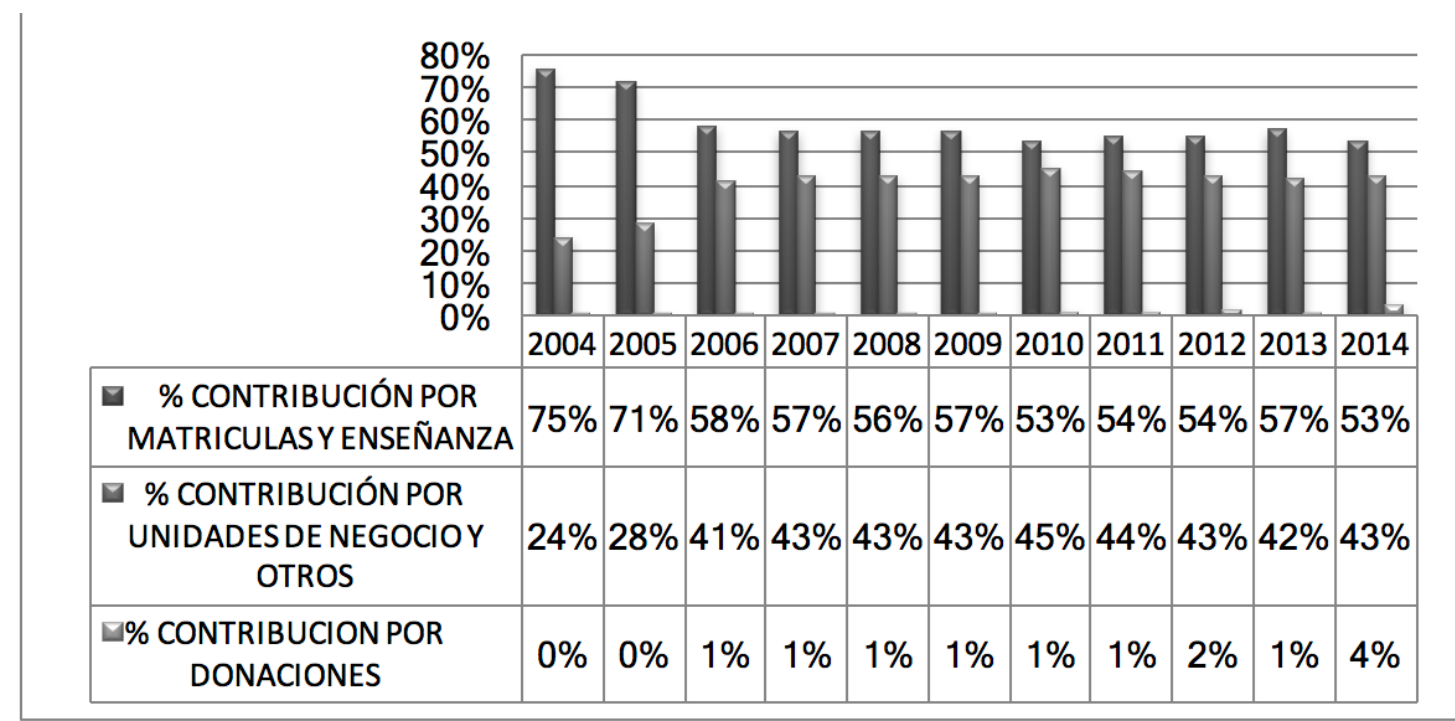

Gráfica 4: Mecanismos de financiamiento IES peruana (2004-2014).

Fuente: Elaboración propia. 
ciero y social, enriqueciendo la misión de la universidad privada que es frecuentemente catalogada de usar la educación para lucrar (Gregorutti, 2011).

Las IES de carácter privado, generan valor social y económico a través de diferentes mecanismos de financiamiento basados en transacciones comerciales y también ajenas a estas. La IES colombiana por medio de las IAS ha contribuido a la financiación de estudiantes de estratos 1,2 y 3; otorgándoles alojamiento, alimentación, trabajo y estudio al interior de la institución. Por su parte, la IES peruana, desarrolla un mecanismo de financiamiento distintivo al de la IES colombiana, porque crea la posibilidad de autofinanciación en dos sentidos: primero bajo condiciones especiales de becas de estudio, la administración de la IAS otorga beneficios de hasta el 33\% en el costo total de las matrículas a los hijos de los empleados de la organización. En relación con los estudiantes que no cumplen esta condición, se les otorga créditos para obtener los productos de las IAS aún precio mínimo, para ser distribuidos de manera informal entre clientes que están dispuestos a pagar el valor asignado a los bienes ofrecidos, con la convicción de que su compra contribuye en parte a suplir esta necesidad social.

En pocas palabras, este modelo ha permitido generar un impacto social importante en por lo menos tres grandes aspectos, a saber: 1) Una mejora en la calidad de vida de los estudiantes, quienes se ven beneficiados por la educación recibida, con un posible ascenso de estrato social al egresar de la institución e insertarse en el mercado laboral; 2) La creación de valor económico a través de los mecanismos de financiamiento que también impactan positivamente la economía de las familias de los estudiantes, que se ven beneficiadas por el auto sostenimiento en favor de sus hijos (Gregorutti, Naranjo \& Marín, 2015).
Las economías regionales que se ven mejoradas por emprendimientos que impulsan más empleo, en el contexto de una creciente mentalidad emprendedora para superar las barreras que impiden el desarrollo.

Como se mencionó en la introducción, las instituciones estatales, tradicionalmente, han dependido de las asignaciones financieras que los diversos presupuestos gubernamentales les asignan. Esta es una alternativa todavía válida, aunque tiende a ser insuficiente para la creciente demanda de educación superior. Una diversificación de los mecanismos de financiamiento puede ser un complemento que ayude a cerrar la brecha.

En el contexto de pobreza que impacta las sociedades de los países latinoamericanos, la promoción y oferta de educación terciaria no necesariamente garantizan su acceso. Muchos de los jóvenes experimentan problemas estructurales que les impiden estudiar aun cuando tengan que pagar una matrícula reducida. En términos de políticas para promover modelos de financiamiento innovadores, se deben considerar la mayor cantidad posible de dimensiones involucradas en la vida de los jóvenes para que el acceso y continuidad sean garantizados. De allí que estos dos casos brindan modelos innovadores que pueden servir para iniciar proyectos similares en otros contextos.

Finalmente, es importante destacar que, si bien es cierto que las IES deben explorar nuevos modos de autofinanciamiento para contribuir directamente en la creación de una sociedad más equitativa y con mejores oportunidades para los menos afortunados, esas contribuciones, al mismo tiempo deben ser enmarcadas desde una misión esencialmente educativa. El tiempo dirá si este balance es posible en una región con tanto potencial como la Latinoamericana. 


\section{Referencias}

Alcántar, V. M., Arcos, J. L. \& Ocegueda, J. M. (2008). Gestión del financiamiento alterno en las instituciones de educación superior: la experiencia de sorteos universitarios en la UABC. Revista de la educación superior, 37, $97-114$.

Alter, S. K. (2006). Social enterprise models and their mission and money relationships. In Social entrepreneurship: New models of sustainable social change. pp. 205-232.

Améstica, L., Gaete, H. \& Llinas-Audet, X. (2014). Segmentación y clasificación de las universidades en Chile: desventajas de inicio y efectos de las políticas públicas de financiamiento. Segmentation and classification of universities in Chile: disadvantages from the start and effects of public financing policies. 22(3), 384-397.

Austin, J. E., Gutiérrez, R., Ogliastri, E., \& Reficco, E. (2006). Gestión efectiva de emprendimientos sociales: Lecciones extraídas de empresas y organizaciones de la sociedad civil en Iberoamérica: Banco Interamericano de Desarrollo.

Banco Mundial (2012). Evaluaciones de políticas nacionales de Educación: La Educación superior en Colombia (9264180710). Retrieved from

Barriga, A. D., Cardiel, H. C., López, R., Maldonado, A. \& Mendoza, J. (1996). Financiamiento y gestión de la educación superior en América Latina y el Caribe: UNAM, Centro de Estudios Sobre la Universidad.

Bernheim, C. T. (2003). La universidad ante los retos del siglo XXI: Ediciones de la Universidad Autónoma de Yucatán.

Casani, F., Pérez-Esparrells, C. \& Rodríguez, J. (2010). Nuevas estrategias económicas en la universidad desde la responsabilidad social. Calidad En La Educación (33), 255-273.

Coraggio, J. L. (2011). La sostenibilidad de los emprendimientos de la economía social y solidaria. Otra Eco- nomía, 2(3), 41-57.

Céspedes, H. (2009). El emprendimiento social y su sustentabilidad. HC Global Group.

De Benito, C. (2008). Crisis económica y crisis ética: Responsabilidad Social Empresarial y virtudes humanas. (Spanish). Capital Humano, 21(227), 62-72.

De Fanelli, A. G. (2007). La reforma universitaria impulsada vía el financiamiento: Alcances y limitaciones de las políticas de asignación. Espacio Abierto. Cuaderno Venezolano De Sociología, 16(1), 7-29.

Demil, B. \& Lecocq, X. (2009). Evolución de modelos de negocio: Hacia una visión de la estrategia en términos de coherencia dinámica. (Spanish). Business Model Evolution: Towards a Dynamic Consistency View of Strategy. (English)(23), 86-107.

Díaz, N. \& Castaño, C. A. (2013). El Valor Compartido como Nueva Estrategia de Desarrollo Empresarial. Daena: International Journal of Good Conscience, 8(2), 82-100.

Díaz, C. \& Fernández, J. L. (2016). Marco conceptual de la ética y la responsabilidad social empresarial: un enfoque antropológico y estratégico. Revista Empresa $Y$ Humanismo, XIX(2), 69-118. https://doi.org/10.15581/015. XIX.2.69-118

Duque, E. (2007). La empresa social y su responsabilidad social. Revista Innovar, 17(30)

Foncea, M. D., Marcuello, C. \& Marcuello, C. (2012). Empresas sociales y evaluación del impacto social. (Spanish). Social enterprises and assessment of their social impact. (English)(75), 179-198.

Gregorutti, G. (2011). Commercialization of higher education in Latin America: The case of Mexico. Comparative \& International Higher Education, 3(1), 11-14.

Gregorutti, G., Naranjo, O., \& Marín, C. (2015). Un estudio comparado del valor económico y social de dos universi-dades adventistas. Valor Agregado, 2, 9-20. 
Lescano, L. (2011). La orientación al servicio, los roles y la formación de los mandos intermedios en las organizaciones de servicio. Pamplona. España: Instituto Empresa y Humanismo - Universidad de Navarra.

Mair, J. \& Noboa, E. (2003). Emergence of Social Enterprises and Their Place in the New Organizational Landscape.

Muñoz, J. (2013). Ética empresarial, Responsabilidad Social Corporativa (RSC) y Creación de Valor Compartido (CVC). Business Ethics, Corporate Social Responsibility (CSR) and Creating Shared Value (CSV). 7(3), 76-88. doi:10.3232/GCG.2013.V7.N3.05

Méndez, J. A., \& Peralta, D. A. (2014). Reflexiones respecto a la responsabilidad social empresarial y la creación de valor económico desde la perspectiva de los proveedores. Reflections on the Corporate Social Responsibility and the Creation of Economic Value from the Point of View of Providers., 15(38), 625-645. doi:10.11144/ Javeriana.cc15-38.rrrs

Molina, V. M., Armenteros, M. \& López, S. (2014). Modelo de negocio e innovación. Experiencia del laboratorio de competitividad empresarial de la PYME. (Universidad Autónoma de Coahulia, Ed.). Mèxico D.F.: Plaza y Valdés Editores.

Naranjo, O. (2015). Propuesta de un modelo de negocio desde el emprendimiento social. Apuntes Universitarios (1), 35-50.

Nicholls, A. (2006). Social entrepreneurship: New models of sustainable social change: Oxford University Press.

Novy-Hildesley, J. (2007). Social entrepreneurship yields high returns. Far Eastern Economic Review, 170(3), 61.

Pazos, D. R., López, S. F., González, L. O. \& Sandiás, A. R. (2008). La creación de empresas en el ámbito universitario: una aplicación de la teoría de los recursos. (Spanish). Firm creation in universities: a resource based view. (English), 8(2), 11-28.

Porter, M. E. \& Kramer, M. R. (2011). Creating shared value.
Harvard business review, 89 (1/2), 62-77.

Rama, C. (2012). El negocio universitario for-profit en América Latina. Revista de la Educación Superior, XLI(4), 60-95.

Ruiz, I., Cano, C. A. \& Montes, I. C. (2008). Financiamiento y demanda de educación superior en Colombia (periodo 1990-2005). Calidad En La Educación (29), 111-136.

Spickett-Jones, J. G., Philip, J. K. \& Jon, D. R. (2004). Social facts and ethical hardware: Ethics in the value proposition. Journal of Communication Management, 8(1), 68-82. doi:10.1108/13632540410807556

Stake, R. E. (1998). Investigación con estudio de casos. Madrid: Ediciones Morata.

Stake, R. E. (2013). Multiple case study analysis. New York: Guilford Press.

Terzolo, M. S. (2014). Aplicación de la teoría de los Grupos de Interés (Stakeholders) en una empresa comercial de la ciudad de Mar del Plata. (Doctoral dissertation, Universidad Nacional de Mar del Plata).

Vásquez, A. G., \& Dávila, M. A. T. (2008). Emprendimiento social - revisión de literatura. (Spanish). Social Entrepreneurship - Literature Review. (English), 24 (109), $105-125$.

Vázquez-Maguirre, M. \& Portales, L. (2014). La empresa social como detonadora de calidad de vida y desarrollo sustentable en comunidades rurales. (Spanish). Social enterprise as a generator of quality of life and sustainable development in rural communities. (English)(37), 255-284.

Young, R. (2006). For what it is worth: Social value and the future of social entrepreneurship. Social entrepreneurship: New models of sustainable social change, 56-73.

2018, Vol. 11(22) 168-183. (T) The Author(s) 2018 Reprints and permission: www.americana.edu.co

https:/www.coruniamericana.edu.co/publicaciones/ojs/index.php/pensamientoamericano/index 